\title{
Supportive treatment - an integral part of cancer care
}

\author{
F. Lordick \\ Department of Medical Oncology and Hematology, Klinikum Braunschweig, Braunschweig, Germany
}

Received 7 December 2011; accepted 16 January 2012

This issue of the Magazine of European Medical Oncology is dedicated to the supportive aspects of cancer care reflecting the increasing knowledge and advances in this field.

There is no doubt that optimal treatment of cancer can only be achieved if the potential side-effects and complications of treatment are known and professionally managed. This is not the task of a "supportive cancer care specialist". It is the genuine task of every physician, irrespective of his subspecialization, to know in depth and detail the possible complications of the treatment he delivers. Patients must be informed about it and instructed how to behave in order to prevent complications. And doctors must know how to react when complications occur. Cancer treatment can be lifethreatening. To give an example: if, during myelotoxic chemotherapy, the patient feels shivering she or he needs to see the doctor as soon as possible in order to get the right antibiotic treatment for febrile neutropenia and to get access to cardiorespiratory observation and support, if necessary. Doctors who failed to inform patients about this complication and to instruct them properly and who are not able to manage febrile neutropenia appropriately must not deliver highly toxic and dangerous chemotherapy regimens like dose-dense adjuvant epirubicine-paclitaxel-cyclophosphamide (ETC) in patients with high-risk breast cancer.

The situation is really not simple in cancer treatment. As multi-disciplinary treatment approaches are increasing, and we need to know more and more not only about our own tools but also about the possibilities, limitations and complications of the treatment delivered by our partners. To give another example: The discussion about the reduction of surgical axillary lymph node dissection in sentinel-node positive and clinically node-negative breast cancer is incomplete and looks even unsound as long as we have not yet defined the role of axillary radiation in this particular situation. If breast surgeons prompt radiation oncologists or leave it up to personal judgment to deliver more treatment to the axilla as compensation for the less radical surgical approach, the outcome for the patients in terms of lymphoedema and other side-effects may be even worse instead of better.

Correspondence: Florian Lordick, MD, Department of Medical Oncology and Hematology, Klinikum Braunschweig, Celler Str. 38, 38114 Braunschweig, Germany.

E-mail: medklinik3@klinikum-braunschweig.de
When cancer has turned into the chronic phase, it is often symptomatic and patients need support and treatment in many aspects of life, ranging from somatic to psychological and spiritual. Our current understanding is that early supportive interventions according to the needs of the individual patients are more effective than late interventions and may even prolong the patients' survival. In some situations like therapy-associated nausea and vomiting, preventive strategies have led to marked improvements of treatment tolerability and quality of life. This should not lead to an unjustified actionism in all fields. Anaemia correction is one of the examples where we had to learn that more is not always better. We need study-relevant endpoints. The over-interpretation of surrogate endpoints like haemoglobin correction during the use of erythropoiesis-stimulating agents was not adequate and patients were harmed when too much haemoglobin correction was attempted. We should talk with the patient about typical cancer-associated symptoms from the beginning and increase the awareness that many symptoms can be managed and effectively relieved when adequate treatment is offered. Cancer-associated pain, chemotherapy-induced vomiting and skin-rash associated with epidermal growth factor receptor (EGFR) directed treatments are good examples.

A deeper understanding of tumour pathogenesis and cancer biology is changing the way how we treat cancer. Gastrointestinal stromal tumours and EGFR-mutated non-small cell lung cancer are two prominent examples. Targeted treatment has also enlarged our options in the supportive care of cancer. The newest developments are the RANK-ligand antibody denosumab which has proven efficacy in the prevention of skeletal related events in cancer metastasized to the bone and the anti-EpCAM and anti-CD3 bispecific monoclonocal antibody catumaxomab. This drug was developed by an academic start-up company from Munich, German and is now approved for an intraperitoneal application in malignant ascites due to epithelial cancer expressing EpCAM. Catumaxomab significantly prolongs the time to the next paracentesis. All physicians who treat patients with malignant ascites that recurs every week know how much this condition impairs life and how much patients benefit if the interval of recurrence can be prolonged.

Cancer causes symptoms, threatens life, changes the patients' self-determination, disrupts the scheme of life and alters the social functioning of the patients, inside and outside their families. Many patients need help in order to cope 
with the extreme challenge that cancer brings into their lives. If the threat that cancer brings into a patient's life is too strong and his or her own resources are too weak or cannot be accessed, anxiety and depression will emerge. Who is the person to detect a patient's distress and desperation? Can we wait until a patient indicates his need for psychological support by himself or herself? Certainly not! Cancer teams have to be trained in order to be sensitive for the patients' mental and emotional needs. Furthermore, we need to systematically screen our patients for their psychological distress. There is an ongoing debate on who should do that and how this should be done. There is an increasing awareness that not only the cancer patient himself or herself but also, and sometimes even more, his relatives and loved-ones suffer from the situation and need to be included into the treatment process. Psycho-oncology is an emerging discipline and we are developing the tools and methods that can effectively be applied in cancer care. One thing seems clear and unquestionable: Empathy and sincereness should always be the mainstay of any psycho-oncological intervention.

This issue sheds light on some of the current aspects of supportive care in cancer. The authors, all well-known experts in their field, should be congratulated for their excellent work and their willingness to participate in this theme issue. 